JOURNAL OF BUSINESS

and entrepreneurial

studies

\title{
Evaluación de la calidad de los servicios hoteleros de segunda categoría del sector céntrico de Guayaquil
}

\section{Evaluation of the quality of the second category hotel services in the downtown area of Guayaquil}

Ing. Nuria Zambrano Camacho, Mgs Docente Universidad Auntónoma de México nuria.zambranocam@gmail.com https://orcid.org/0000-0002-3775-6763

Ing. Janina Arteaga Cisneros, Mgs Universidad San Marcos janina.arteagaci@hotmail.com https://orcid.org/0000-0001-6749-6088

Recibido 23 enero 2017 - Aceptado junio - 2017 


\section{Resumen}

La presente investigación trata de la evaluación de la calidad de los servicios en los establecimientos hoteleros de segunda categoría del sector céntrico de la ciudad de Guayaquil, con el objetivo de determinar si existen falencias en el servicio para mejorar la gestión de sus elementos tangibles e intangibles que inciden en la satisfacción de sus usuarios.

De igual forma se recopilo información por medio de encuestas, lo que permitió conocer la situación actual de los hoteles de segunda categoría así como la percepción que tienen sus usuarios referentes al cumplimiento de sus expectativas $\mathrm{y}$ proponer planes de mejora.

Palabras clave: calidad, gestión, mejora, satisfacción, usuarios.

\begin{abstract}
This research is about the quality of services in the hotel establishments of second category of the central area at Guayaquil city, in order to determine whether there are gaps in service to improve the management of its tangible and intangible elements that affect user satisfaction. Likewise, information was collected through surveys, allowing the current situation of second-class hotels as well as the perception its users concerning the fulfillment of their expectations with the objective to propose an improvement plan.
\end{abstract}

Keywords: quality, management, improvement, satisfaction, users.

\section{1.- INTRODUCCIÓN}

Cada día miles de personas viajan internamente o fuera de sus países con fines comerciales, recreativos $\mathrm{o}$ de estudio. En el ecuador sólo en el periodo de enero a septiembre del 2014 el ingreso por divisas de turismo registró un valor de $\$ 1.085,5$ millones con un crecimiento del $23 \%$ en relación al mismo periodo del año anterior. (Ministerio de Turismo, 2014)

El actual gobierno a través de la matriz productiva busca impulsar el turismo como uno de los sectores estratégicos a desarrollar. Para lograr lo antes expuesto la industria turística deberá ser capaz de proveer servicios básicos de calidad que cumplan con las expectativas de sus usuarios.

Referente a los servicios de los establecimientos hoteleros, estos deberán brindar según Gandara (s.f) citado en Zambrano (2015) "servicios e instalaciones de buena calidad y una relación calidad-precio satisfactoria" en función de su categoría. Para lograr lo antes expuesto, los hoteles deberán tener un sistema de calidad que les permita gestionar correctamente sus recursos tangibles e intangibles con la finalidad de superar las expectativas de sus clientes a través de un óptimo servicio encaminado a la excelencia.

Sin embargo en un análisis exploratorio se evidencio que los hoteles de segunda categoría de la ciudad de Guayaquil, al tener una estructura organizacional tipo familiar no cuentan con un sistema de calidad acorde a su categoría lo que podría incurrir en la insatisfacción de sus usuarios. Por lo que el presente estudio pretende diagnosticar la percepción que 
tienen los usuarios sobre estos establecimientos hoteleros en cuanto a la infraestructura y personal de los hoteles para conocer su incidencia en la satisfacción de los mismos.

\section{2.- MATERIALES Y MÉTODOS}

\section{Marco Metodológico}

Dentro de la metodología tenemos la formulación del problema referente a si $¿$ Los recursos tangibles e intangibles de los hoteles de segunda categoría de la ciudad de Guayaquil inciden en la satisfacción de sus clientes?

Para contestar lo antes expresado surge la hipótesis sobre la gestión operativa y Administrativa de los recursos tangibles e intangibles de los hoteles de segunda categoría de la ciudad de Guayaquil inciden en la satisfacción de sus usuarios.

Dentro de las variables que conforman dicha hipótesis tenemos:

Variable independiente: Modelo de gestión. (Causa). La misma que será medida bajo las siguientes dimensiones

$>$ Infraestructura del hotel, habitaciones y áreas comunes

$>$ Limpieza de habitaciones y aseo de instalaciones

$>$ Equipamiento de las habitaciones

$>$ Condiciones de Seguridad

$>$ Personal capacitado y amable

$>$ Trato personalizado

Variable dependiente: Satisfacción (Efecto)

La variable Calidad de atención, será medida bajo las siguientes dimensiones:

$>$ Resolución de problemas

$>$ Expectativas satisfechas

$>$ Fidelidad del cliente

Método
El método aplicado es de enfoque mixto (cualitativo y cuantitativo) ya que para esta investigación se tomó fuentes primarias y secundarias. Referente a las fuentes primarias se utilizó la observación y encuesta, las mismas que se realizaron a los usuarios de los hoteles de segunda categoría del sector céntrico de la ciudad de Guayaquil para conocer la percepción de ellos sobre los servicios brindados. En relación a las fuentes secundarias se utilizaron bases de datos tomados del Ministerio de Turismo y de la Federación Hotelera del Ecuador.

\section{Tipo de Investigación}

Para conocer la situación de los establecimientos hoteleros de segunda categoría en la ciudad de Guayaquil, se hizo una investigación Pre-experimental ex-post-facto en dichos hoteles, caracterizada por el nulo o poco control de las variables así como estableciendo relaciones entre las mismas.

Población y muestra

Como población tenemos el número de turistas diarios que pernotan en los diez establecimientos hoteleros de segunda categoría del sector céntrico de la ciudad de Guayaquil. Teniendo un aproximado de 10 usuarios por día por hotel dentro del lapso de una semana lo que dio como resultado 385 huéspedes.

Tabla 1. Hoteles de segunda categoría sector céntrico.

\begin{tabular}{|c|c|c|c|}
\hline Nombre & Dirección & Categoría & Habitaciones \\
\hline NEVADA & $\begin{array}{l}\text { LORENZO DE GARAYCOA } 710 Y \\
\text { QUISQUIS }\end{array}$ & SEGUNDA & 59 \\
\hline $\begin{array}{l}\text { NUEVO } \\
\text { ECUADOR }\end{array}$ & $\begin{array}{l}\text { PEDRO MONCAYO } 1117 \text { ENTRE } \\
\text { AGUIRRE Y LUQUE }\end{array}$ & SEGUNDA & 55 \\
\hline CALIFORNIA & XIMENA 601 Y URDANETA & SEGUNDA & 52 \\
\hline $\begin{array}{l}\text { PLAZA } \\
\text { CENTENARIO }\end{array}$ & $\begin{array}{l}\text { LORENZO DE GARAYCOA } 931-933 \\
\text { ENTRE } 9 \text { DE OCTUBRE Y VELEZ }\end{array}$ & SEGUNDA & 55 \\
\hline CENTENARIO & $\begin{array}{l}\text { VELEZ } 726 \text { Y LORENZO DE } \\
\text { GARAYCOA }\end{array}$ & SEGUNDA & 47 \\
\hline CAPRI & LUQUE 1221 Y MACHALA & SEGUNDA & 58 \\
\hline LA TORRE & $\begin{array}{l}\text { CHILE } 333 \text { Y LUQUE EDIFICIO } \\
\text { TORRE AZUL PISO } 13,14,15\end{array}$ & SEGUNDA & 46 \\
\hline INDIRA & GARCIA AVILES 101 Y P.ICAZA & SEGUNDA & 71 \\
\hline AVENTURA & $\begin{array}{l}\text { GOMEZ RENDON \# } 3122 \text { Y LA } \\
\text { NOVENA }\end{array}$ & SEGUNDA & 37 \\
\hline MALECÓN INN & SUCRE 203 Y PICHINCHA & SEGUNDA & 35 \\
\hline
\end{tabular}


Fuente: Zambrano (2015). Adaptado del Catastro de Alojamiento de Guayaquil del Ministerio de Turismo

Para determinar el tamaño de la muestra se utilizó la fórmula de muestreo proporcional al tener una población finita. Para el cálculo de dicha muestra se tomó como error estándar máximo un $5 \%$ y un nivel de confianza de $95 \%$ dando como resultado una muestra de 193 usuarios. Sin embargo para tener una información más precisa se aproximó a una muestra de 200 encuestas.

\section{3.- ANÁLISIS DE RESULTADOS}

La encuesta constó de 29 preguntas estructuradas de carácter cuantitativa, la misma que se dividió en tres partes. La primera parte referente a los datos de los usuarios, su motivación de viaje y días de permanencia, la segunda parte concerniente a la infraestructura del hotel, habitaciones y áreas comunes y la última parte en relación al personal del hotel. Con esta encuesta se quiso conocer el grado de satisfacción de los usuarios con respecto a los recursos tangibles e intangibles y determinar la brecha existente entre las expectativas previas al consumo con el servicio ofrecido.

De la encuesta realizada se determinó que el $65 \%$ de los usuarios eran de género masculino y el $35 \%$ restante era femenino. De estos usuarios el $68 \%$ eran turistas ecuatorianos y el $33 \%$ extranjeros.

Referente a su motivación para viajar y pernotar en los hoteles antes mencionados, el $44 \%$ lo hizo por vacaciones mientras que el $32 \%$ fue por negocios y un $33 \%$ por otras razones. Los días de permanencia de los huéspedes en los hoteles fueron de 2-3 días con el $42 \%$ en su mayoría con un número de personas que pernotaron en la misma habitación de 2,03 en promedio, siendo la mayoría de los huéspedes profesionales seguidos por comerciantes y estudiantes.

La segunda parte de la encuesta trataba de identificar la percepción que tienen los usuarios sobre la infraestructura y equipamiento de la habitación, así como el estado de las áreas comunes y accesibilidad a las mismas. Aquí se pudo evidenciar que estas no eran del todo del agrado de los huéspedes ya que al hacer mención sobre el estado del mobiliario de la habitación, el $68 \%$ de los huéspedes afirmó estar parcialmente de acuerdo sobre el buen estado del mobiliario, seguido por un $26 \%$ que estuvo de acuerdo y un $7 \%$ en desacuerdo.

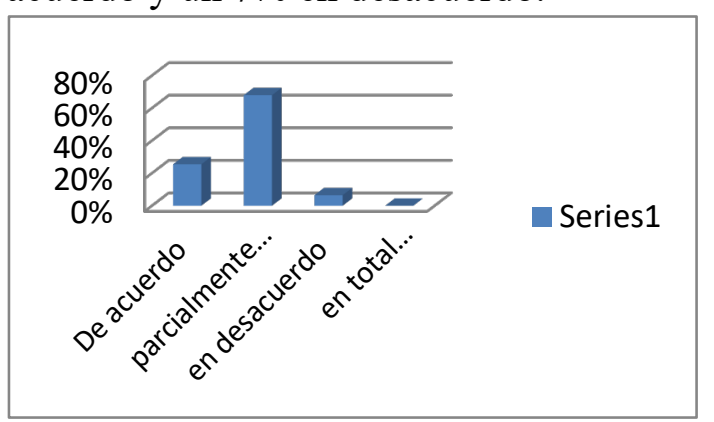

Figura 1. Mobiliario de la habitación Fuente: Zambrano, (2015)

Con respecto al aseo de la habitación se observó que sólo el 26\% de los huéspedes lo catalogó como muy 


\section{JOURNAL OF BUSINESS \\ and entrepreneurial \\ studies}

bueno seguido por un $38 \%$ que lo consideraron bueno y un $37 \%$ como regular. Es decir que uno de los principales elementos que incide en la satisfacción del huésped presenta una fuerte falla al no aplicarse un control eficiente ni procesos de limpieza que garanticen el aseo de las habitaciones al momento de la salida del huésped como ingreso de nuevos huéspedes.

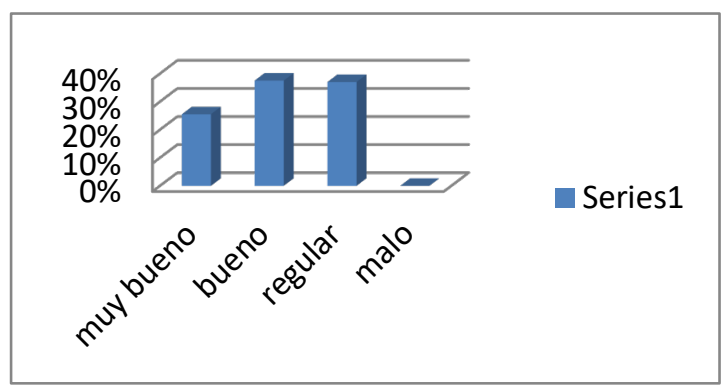

Figura 2. Aseo habitaciones

Fuente: Zambrano, (2015)

Referente a la infraestructura interna y externa de la habitación, se obtuvo que un $47 \%$ lo consideraban bueno, seguido por un $34 \%$ que lo catalogaron como regular y sólo el 19\% como muy buena contrastado con el $1 \%$ que le pareció mala.

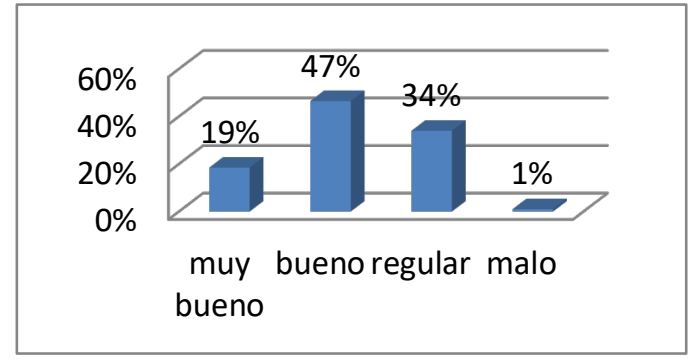

Figura 3. Infraestructura habitación Fuente: Zambrano, (2015)
Un escenario similar se aprecia sobre el equipamiento de la habitación, en la que el $65 \%$ de los huéspedes mencionó estar parcialmente de acuerdo con respecto a si ésta les brindaba comodidad, seguido por un $28 \%$ que estuvo de acuerdo y un $8 \%$ final en desacuerdo.

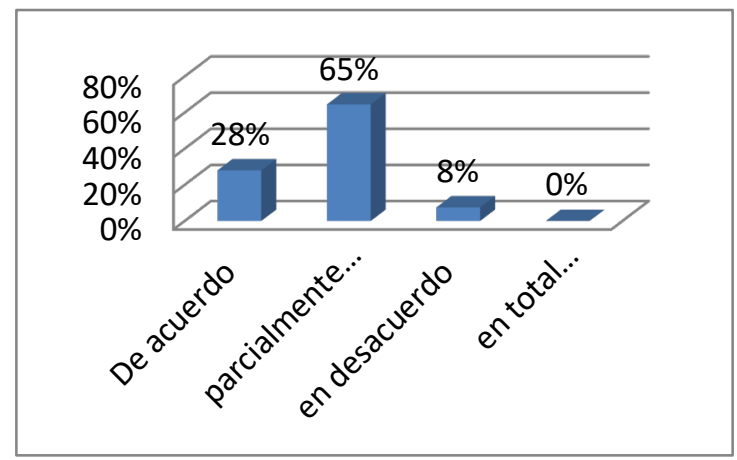

Figura 4. Equipamiento de la habitación Fuente: Zambrano, (2015)

De igual forma en lo que se refiere al mantenimiento e imagen de las áreas comunes como pasillo, escalera y lobby, se evidenció que el $55 \%$ de usuarios lo catalogaron como regular, seguido por el $40 \%$ que lo catalogó como bueno y sólo un $5 \%$ como muy bueno. 
Evaluación de la calidad de los servicios hoteleros de segunda categoría del sector céntrico de Guayaquil

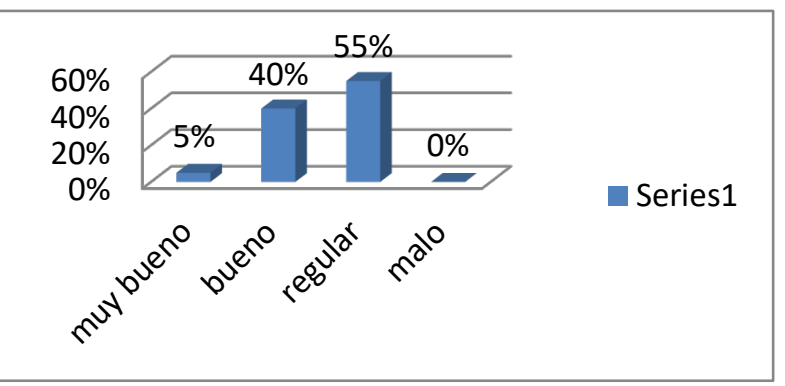

diferencia del $69 \%$ que afirmó no estar de acuerdo.

Figura 6. Profesionalismo de

Recepcionistas

Fuente: Zambrano, (2015)

Esto se puede deber al hecho de que el personal del hotel no disponía de un uniforme estándar, lo que dio como

Figura 5. Mantenimiento en áreas comunes

Fuente: Zambrano, (2015)

Es decir que en lo que se refiere a la infraestructura general del hotel como las habitaciones $\mathrm{y}$ áreas comunes, consideradas como sus recursos tangibles los huéspedes no están del todo satisfecho con las mismas. Es decir, sus expectativas no fueron superadas puesto que la infraestructura interna del hotel presentaba condiciones de deterioro. Así como como una deficiente limpieza de las habitaciones e instalaciones al encontrarse sucias en su mayoría.

Con respecto al personal de recepción se evidenció que sólo el $26 \%$ de los huéspedes consideraban que los recepcionistas demostraban profesionalismo en sus actividades a

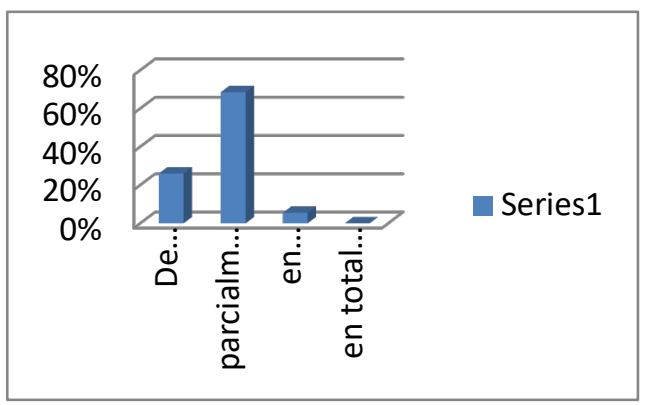
resultado que el $69 \%$ de los huéspedes estuviera en desacuerdo sobre la buena apariencia del personal. Además el 68\% de ellos indicó que hubo falencia en la resolución de problemas mientras que solo un $27 \%$ afirmó estar de acuerdo en que el personal de recepción mostró interés por ayudarlos.

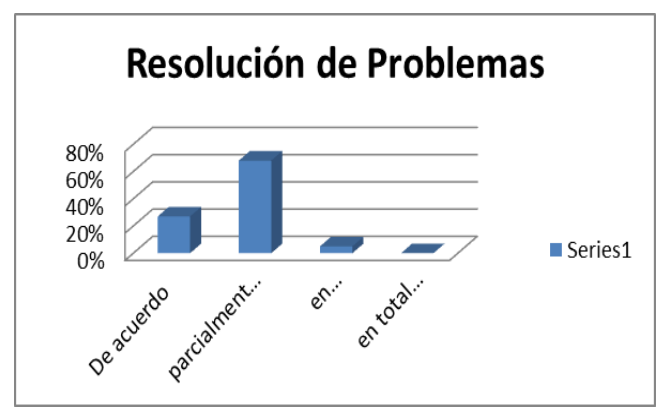

Figura 7. Resolución de Problemas Fuente: Zambrano, (2015)

Esto dio como resultado que el $63 \%$ este parcialmente de acuerdo en que se sus expectativas hayan sido cumplidas. Según Zambrano (2015) los turistas indicaron que "con respecto a la infraestructura el ascensor no funcionaba porque estaba en reparación, que las escaleras estaban un poco 


\section{JOURNAL OF BUSINESS \\ and entrepreneurial \\ studies}

ISSN: 2576-097

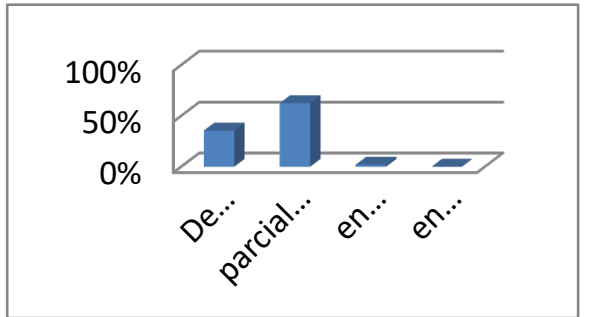

descuidadas, que no había parqueaderos y se sentían inseguros al salir del hotel en la noche". Además mencionaron que el personal del hotel debía ser más amables, $\mathrm{y}$ tener una mejor apariencia.

Figura 8. Cumplimiento de expectativas

Fuente: Zambrano, (2015)

Por lo que al preguntarles si regresarían a dichos hoteles y los recomendarían solo el $40 \%$ lo afirmó que estaría de acuerdo en hacerlo mientras que el $55 \%$ estaba parcialmente de acuerdo y el 5\% en total desacuerdo. Esto denota que dichos usuarios en su próxima visita a Guayaquil no utilizarían estos hoteles. Esto es alarmante ya es más 5 veces más fácil mantener un cliente que captar uno nuevo.

Kotler, P. y Amstrong, G. 2013).

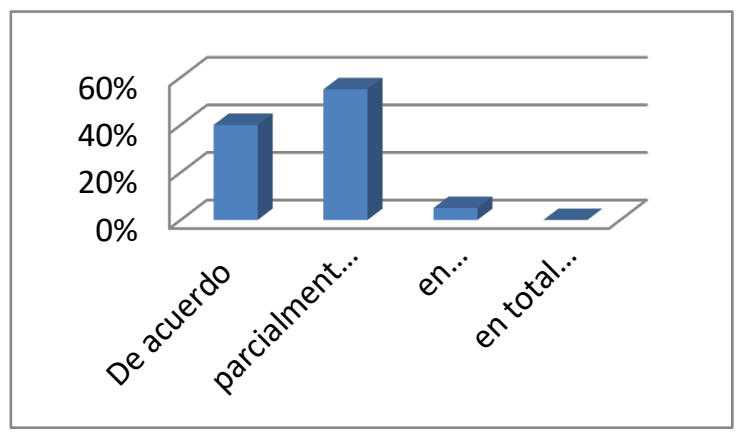

Figura 9. Retorno del huésped al hotel
Fuente: Zambrano, (2015)

\section{4.- DISCUSIÓN}

Con este estudio se evidenció que los establecimientos hoteleros de segunda categoría del sector céntrico de la ciudad de Guayaquil están presentando fallas en la gestión de sus recursos tangibles e intangibles al tener un $63 \%$ de turistas que indicaron que sus expectativas fueron parcialmente cumplidas.

La satisfacción de dichos usuarios o turistas cobra vital importancia para el actual Gobierno que busca a través de su matriz productiva impulsar el desplazamiento de turistas al Ecuador. Para lograr lo antes expuesto estos hoteles deberán ser capaces de cumplir con las expectativas de sus huéspedes teniendo en claro que la satisfacción e intención de recompra de los mismos dependerá de una serie de atributos que componen la vivencia del huésped. Los mismos que van desde la percepción de la infraestructura al momento de su llegada hasta el trato que recibe del personal durante su estadía hasta su partida. Es decir que para que haya satisfacción de los huéspedes referente al servicio de los hoteles, debe haber calidad en los mismos.

Los turistas son cada vez más exigentes con relación a sus necesidades y esperan servicios e instalaciones de buena calidad y una relación calidad-precio satisfactoria, pues su visión holística y globalizada, desarrollada en la sociedad de la información y de las nuevas tecnologías, les permite demandar nuevos productos $\mathrm{y}$ destinos más elaborados y diversificados. Por lo que según Benítez (2010) "se hace más necesario que se dispongan de herramientas de análisis adecuadas para analizar la calidad de servicio" ya que es 
un factor clave para desarrollar estrategias que permitan incrementar la competitividad del destino turístico.

La calidad no es otra cosa que igualar o superar las expectativas que tiene el cliente previo al consumo del servicio. Por lo que se propone un plan de mejoras que constituye las pautas a seguir a fin de que todos los elementos que intervienen en el servicio y que son clave puedan ser sistematizados para su mejor control. Todo esto con la finalidad de mejorar sus resultados en áreas de interés, reduciendo las fallas en las que se incurre. Lo que generará que estos hoteles sean más competitivos al minimizar los costos por no calidad.

El logro de satisfacción de los turistas que pernotan en los hoteles de segunda categoría de Guayaquil dependerá de la calidad de sus servicios y de la existencia de todo un sistema de valores tanto personales como organizacionales comprometidos hacia la excelencia del servicio.

\section{REFERENCIAS}

Benitez, J. (2010). La calidad del servicio en la industria hotelera. (Tesis Doctoral, Universidad de las Palmas de Gran Canaria). Recuperado de: http://acceda.ulpgc.es/bitstream/ 10553/5516/1/0628694_00000_ 0000.pdf

Cámara de Turismo de Pichincha. (2014). Boletín estadístico diciembre 2014. Recuperado de http://www.captur.travel/web2011 /estadisticas turisticas/estadistica. html

Chávez, K y Castro, E (2014). Análisis de la influencia de la calidad del servicio, en la satisfacción de los clientes, en los hoteles de 3 estrellas en la ciudad de guayaquil para diseñar un programa de mejoras en la calidad de servicio al cliente. Recuperado de http://repositorio.ucsg.edu.ec/bitstre am/123456789/2305/1/T-UCSGPRE-ESP-AETH-197.pdf

Coordinación General de Estadísticas e Investigación del Ministerio de Turismo (2014). Ingreso de divisas por turismo creció en un $21 \%$ hasta septiembre del 2014. Recuperado de http://www.turismo.gob.ec/ing reso-de-divisas-por-turismocrecio-en-un-21-hastaseptiembre-del-2014/

Crosby, P. (1987). La calidad no cuesta. El arte de cerciorarse de la calidad. México: CECSA.

El Tiempo (2012). Ecuador busca turismo certificado. Recuperado de

http://www.eltiempo.com.ec/noti cias-cuenca/112052-ecuadorbusca-turismo-certificado

Evans, James y Lindsay, W. (2008). Administración y Control de Calidad. Mexico: Cengage Learning. Recuperado de http://books.google.com.ec/book s? id=bL7PKYd4ypQC\&printsec $=$ frontcover\&hl $=$ es\&source $=\mathrm{gbs}$ ge_summary_r\&cad $=0 \# \mathrm{v}=$ onep age \&q\&f=false

Ministerio de Industria, Energía y Turismo. (s.f). Manual Práctico de Calidad Hotelera para Hoteles y alojamientos rurales. Recuperado de 
http://www.alojamientosconectados.es/t urismo/sites/default/files/10.\%20Calida d\%20Hotelera.pdf

Ministerio de Turismo (2014). Principales Indicadores de Turismo. Boletín N.12. Recuperado de http://www.optur.org/estadistica s/Diciembre_boletin_2014.pdf

Morillo, M (2011). Indicadores de gestión de la calidad de los servicios de alojamiento turístico del estado de Mérida. Actualidad Contable Faces, (14) 22, 86-119. Recuperado de http://www.redalyc.org/articulo. oa? id $=25720061007$

Llamas, C. (2008). Gestión de alojamientos turísticos. España: Liber Factory.

Organización Mundial de Turismo (2013). Tendencias del Turismo y Estrategias de Marketing OMT. Barómetro del turismo mundial, 11, (1-6).

Organización Mundial de Turismo (2010). Normas y sistemas de calidad en el turismo y su relación con la sostenibilidad y leyes de turismo. La experiencia de las Américas. Recuperado de http://www.siimt.com/work/sites /siimt/resources/LocalContent/1 172/6/normsiscalturi_protec.pdf

Padrón, V. (2002). Sistemas de calidad aplicados a los establecimientos hoteleros. Recuperado de http://www.ulpgc.es/hege/almac en/download/4/4373/MODULO 3.pdf

Plan Nacional del Buen Vivir. 20132017. Objetivos del Plan Nacional del Buen Vivir. Recuperado de http://www.buenvivir.gob.ec/her ramientas

Reglamento General de Actividades Turísticas. Decreto No. 3400. Recuperado de http://www.hotelesecuador.com. ec/downloads/Reglamento\%20G eneral $\% 20 \mathrm{de} \% 20$ Actividades $\% 2$ 0Turisticas.pdf

Tari, J. y Pereira, J (2012). Calidad y rentabilidad. Análisis del certificado Q en las cadenas hoteleras. Universia BusinessReview. Recuperado de http://rua.ua.es/dspace/bitstream/ 10045/27964/1/2012 Universia BusinessReview.pdf. 